OPEN ACCESS

Edited by:

Alexandre Andrade Loch, University of São Paulo, Brazil

Reviewed by: Yutong Jiang,

Third Affiliated Hospital of Sun Yat-sen University, China Daniela Adamo, University of Naples Federico II, Italy

*Correspondence:

Zhongyuan Xia xiazhongyuan2005@aliyun.com Shaoqing Le leishaoqing@163.com

these authors have contributed equally to this work

Specialty section: This article was submitted to Public Mental Health, a section of the journal Frontiers in Psychiatry

Received: 05 May 2020 Accepted: 13 July 2020 Published: 22 July 2020

Citation:

Wang W, Song W, Xia Z, He Y, Tang L,

Hou J and Lei S (2020) Sleep Disturbance and Psychological

Profiles of Medical Staff and Non-Medical Staff During the Early Outbreak of COVID-19 in Hubei

Province, China.

Front. Psychiatry 11:733 doi: 10.3389/fpsyt.2020.00733

\section{Sleep Disturbance and Psychological Profiles of Medical Staff and Non- Medical Staff During the Early Outbreak of COVID-19 in Hubei Province, China}

\author{
Wei Wang ${ }^{1 \dagger}$, Wenqin Song ${ }^{1 \dagger}$, Zhongyuan Xia ${ }^{1 *}$, Yuhong $\mathrm{He}^{2}$, Linghua Tang $^{1}$, Jiabao Hou ${ }^{1}$ \\ and Shaoqing $\mathrm{Lei}^{1 *}$ \\ 1 Department of Anesthesiology, Renmin Hospital of Wuhan University, Wuhan, China, ${ }^{2}$ Office of Infection Control, Renmin
Hospital of Wuhan University, Wuhan, China
}

Objective: The outbreak of coronavirus disease 2019 (COVID-19) has considerably burdened the healthcare system in the Hubei Province, the most severely affected region in China. The aim of our study was to assess the psychological effects of COVID-19 epidemic on the healthcare workers in Hubei.

Methods: A total of 2737 healthcare workers were sampled using a two-dimensional code shared online between Mar 4 and Mar 9, 2020. The questionnaires consisted of three elements: baseline characteristics, Pittsburgh Sleep Quality Index (PSQI), and Hospital Anxiety and Depression Scale (HADS). The primary outcome variables were PQSI, anxiety and depression scores of non-medical staff, non-frontline medical staff and frontline medical staff. Binary logistical regression analyses were used to compare between respondents with and without sleep disturbance.

Results: About $61.6 \%$ of the respondents reported sleep problems, 22.6\% experienced anxiety, and 35\% exhibited depressive symptoms. The prevalence of sleep disorders was higher among the frontline healthcare workers compared to the non-frontline and nonmedical staff, while anxiety and depression were prevalent in the entire cohort. Logistic regression analysis identified medical occupation, family burden, bereavement, anxiety, and depression as significantly predictive of poor sleep quality.

Conclusions: Frontline medical staff are more vulnerable to sleep disturbances. Psychosocial interventions are needed to help allied healthcare personnel to better respond to COVID-19 and future outbreaks.

Keywords: Coronavirus Disease 2019, Pittsburgh sleep quality index, Hospital Anxiety and Depression Scale, frontline medical workers, Mental health, public health emergency, pandemic 


\section{INTRODUCTION}

The first case of coronavirus disease 2019 (COVID-19) was reported in December 2019 in Wuhan, Hubei province, China. It is caused by the severe acute respiratory syndrome coronavirus 2 (SARS-CoV-2) $(1,2)$, which is transmitted through respiratory droplets and direct human contact, as well as via asymptomatic carriers $(3,4)$. The epidemic rapidly spread from Hubei to other parts of China since an estimated five million people had travelled from Wuhan during the Spring Festival (5). To curb the transmission of COVID-19, the Chinese government imposed quarantine in Wuhan and Hubei since Jan 23-24, 2020. Only one person from each household is permitted to shop for provisions once every two days, except for medical reasons. Furthermore, 19 provinciallevel regions have extended logistical support to different areas of Hubei. According to the National Health Commission of China, over 42,000 healthcare workers in 345 medical assistance teams from across the country are in Hubei to control the epidemic as of Mar 8, 2020 (6).

SARS-CoV-2, also known as 2019 novel coronavirus (2019-nCOV), has mainly infected the 30-79 years age group $(7,8)$, of which $3.5-3.8 \%$ are healthcare workers $(8$, 9). The frontline medical staff must wear enhanced droplet/ airborne personal protective equipment, including fit-tested N95 mask, fluid-resistant gown and eye protector, which puts them at a high risk of physiological and psychological problems (10). There were reports of fatigue, poor sleep, fear, anxiety, depression and even post-traumatic stress symptoms (PTSD) among the frontline healthcare workers during the SARS and Ebola outbreaks (11-13). COVID-19related-PTSD has been shown to be positively correlated with sleep disturbance, anxiety and depression in Chinese and Italian populations, as well as worsening the quality of life of healthcare workers and patients. $(14,15)$. The psychological intervention medical team from the Second Xiangya Hospital of Central South University, Hunan Province, China found that several nurses serving COVID-19 patients reported excitability, agitation and insomnia (16). Qiu D et al. conducted a systematic review and meta-analysis which showed that allied medical workers are more vulnerable to sleep disturbances compared to the general population in China (17). A multi-center study conducted from late January to early February, 2020 reported a significant correlation between frontline medical jobs and mental health issues (18). It was not until late February 2020 that the shortage of masks, health equipment, physicians and nurses in the Hubei province was effectively addressed. A positive trend in the epidemic prevention and control has emerged in March 9 (19). There is still uncertainty, nevertheless, whether consideration of early March data changes the conclusions regarding the psychological effect of COVID-19 on medical staff and non-medical staff.

In the present study, we analyzed the sleep quality, and the levels of anxiety and depression among the medical and non- medical workers in Hubei province, the most seriously affected region in China in order to determine the psychological effects of the outbreak.

\section{METHODS}

\section{Study Design}

The present study was reviewed and approved by the ethic committee of Renmin Hospital of Wuhan University and registered at the Chinese Clinical Trial Registry (ChiCTR2000030985). An anonymous cross-sectional survey was conducted on the medical staff (also referred to as healthcare workers, composed of doctors and nurses) of multiple hospitals and non-medical staff (students, community workers, volunteers, etc.) in the Hubei Province from March 4 to 9, 2020 using an online questionnaire posted as a two-dimensional code. Eligible participants were men and women aged 18-65 years old who had electronic social software. The subjects were instructed to share their responses on WeChat Moments or via Email.

\section{Questionnaires}

Questions regarding demographic information such as age, gender, marital status, education, locations in February, occupation during the epidemic and comorbidities (cardiovascular diseases [categorized into hypertension, diabetes, and coronary heart disease], liver and kidney diseases, etc.), sleep quality, anxiety, and depression were included in the questionnaire.

The Pittsburgh Sleep Quality Index (PSQI) score was devised 30 years ago by Buysse et al. to assess multiple factors related to sleep quality. The questionnaire comprises of 19 individual items in seven domains: subjective sleep quality, sleep latency, sleep duration, habitual sleep efficiency, sleep disturbances, use of sleep medication, and daytime dysfunction. Each domain score ranges from 0 to 3 and the total scores from $0-21$, with higher scores indicating lower sleep quality. A total PSQI score greater than 5 indicates poor sleep with a diagnostic sensitivity of $89.6 \%$ and specificity of $86.5 \%$ (20). In addition, subjects with PSQI $>10$ are considered bad sleepers (20).

The Hospital Anxiety and Depression Scale (HADS) is a 14item self-administered questionnaire that consists of 7 items for anxiety scale (HADS-A) and 7 items for depression (HADS-D) (21). Each item is rated on a 4-point Likert scale (0-3) and higher scores indicate greater anxiety and depression severity. A score of 8 or more represents probable case of depression (21).

\section{Statistical Analysis}

Statistical analysis was performed using SPSS Statistics version 16.0 software (IBM). Continuous variables were compared using two-tailed $t$ tests or Mann-Whitney tests depending on data distribution. Chi-square or Yates' continuity corrected chisquare tests were used to compare categorical variables. Associations between risk factors and poor sleep quality were determined with binary logistic regression analysis. $\mathrm{P}<0.05$ was considered statistically significant. 


\section{RESULTS}

\section{Respondent Characteristics}

We received responses from 2,777 participants, and 2,737 (98.6\%) completed the questionnaires, of which 20 did not conform to the inclusion criteria, 10 contained invalid information and 706 were from outside Hubei Province. For the remaining 2,001 respondents, the median age was 33 years (IQR, 28-40) and median BMI was 22 (IQR, 20.0-24.0). The overwhelming majority (95.7\%) of these respondents had more than 12 years of education. In addition, 1,291 (64.5\%) respondents were female, 1,419 (70.9\%) were married, 1,213 (60.6\%) were aged 18-35 years, 126 (6.3\%) had underlying comorbidities, 444 (22.2\%) reported shortage of supplies, 301 (15.0\%) had a COVID-19 patient in their family, and $39(1.9 \%)$ were confirmed or highly suspected of COVID-19. Only 673 (33.6\%) respondents were from Wuhan, and the others (66.4\%) from other cities in Hubei Province, China (Table 1). In terms of occupation, 1,514 (75.7\%) were healthcare workers, including 661 frontline medical professionals. Compared to non-healthcare workers, the healthcare workers were older, female, married, had higher education level, and were more likely to be under pressure of caring for the elderly or children (Table 1). Frontline medical workers were older and had higher BMI as compared to the non-frontline workers. More male healthcare workers were in the frontline rather than non-frontline jobs (268[40,5\%] vs. $249[29.2 \%]$ ). Among the frontline healthcare workers, 418 (63.2\%) had the burden of caring for the elderly or children, and 73 (11\%) had recent COVIDrelated bereavement. These percentages were higher than that for nonfrontline healthcare workers. There were no significant differences in the number of smokers and alcohol use between the frontline and nonfrontline healthcare workers (Table 1).

\section{Effects of the COVID-19 Epidemic on Sleep, Anxiety, and Depression in Frontline Medical Workers}

The median (interquartile range, IQR) PSQI, HADS-A and HADS-D scores of the overall cohort were 7 (4-9), 5 (2-7), and 6 (3-9), respectively. The frontline healthcare workers scored higher for all 3 scales compared to the non-medical staff and non-frontline healthcare workers (Figures 1 and 2A). PSQI component scores for sleep quality, sleep duration, habitual sleep efficiency and daytime dysfunction in frontline healthcare workers were significantly higher than that of non-frontline workers and non-medical staff (Figures 2B, D, E, H). Medical staff reported higher sleep latency and sleep disturbance scores, compared with non-medical staff (Figures 2C, F). There was

TABLE 1 | Demographics characteristics of study participant.

\begin{tabular}{|c|c|c|c|c|c|c|c|}
\hline & $\frac{\text { Overall cohort }}{(n=2 \text { 001) }}$ & \multicolumn{2}{|c|}{ Medical staff } & $\mathbf{P}^{\mathrm{a}}$ value & \multicolumn{2}{|c|}{ Frontline medical workers } & $\mathbf{P}^{\mathrm{b}}$ value \\
\hline Age(y) & $33(28-40)$ & $31(26-37)$ & $33(29-40)$ & $<0.01$ & $33(29-40)$ & $34(30-40)$ & 0.01 \\
\hline $36-44$ & $480(24.0)$ & $93(19.1)$ & $387(25.6)$ & $<0.01$ & 199(23.3) & $188(28.5)$ & 0.02 \\
\hline $45-65$ & $308(15.4)$ & $59(12.1)$ & $249(16.4)$ & 0.02 & 139(16.3) & $110(16.6)$ & 0.86 \\
\hline Gender & & & & 0.03 & & & $<0.01$ \\
\hline \multicolumn{8}{|l|}{ Marital status } \\
\hline Unmarried & $534(26.7)$ & 194(39.8) & $340(22.4)$ & $<0.01$ & $191(22.4)$ & $149(22.5)$ & 0.94 \\
\hline Married & $1419(70.9)$ & $276(56.7)$ & $1143(75.5)$ & $<0.01$ & $646(75.7)$ & $497(75.2)$ & 0.81 \\
\hline Divorced or Widowed & 48(2.4) & $17(3.5)$ & $31(2.1)$ & 0.07 & 16(1.9) & $15(2.2)$ & 0.59 \\
\hline Education level & & & & $<0.01$ & & & 0.95 \\
\hline$\leq 12$ years & $86(4.3)$ & $72(14.8)$ & $14(0.9)$ & & $8(0.9)$ & $6(0.9)$ & \\
\hline Region & & & & 0.03 & & & 0.11 \\
\hline Wuhan & 673(33.6) & 184(37.8) & 489(32.3) & & 261(30.6) & 228(34.5) & \\
\hline Hubei province outside Wuhan & $1328(66.4)$ & $303(62.2)$ & $1025(67.7)$ & & $592(69.4)$ & $433(65.5)$ & \\
\hline COVID-19 diagnosed or suspected & $39(1.9)$ & $4(0.8)$ & $35(2.3)$ & 0.04 & $15(1.8)$ & $20(3.0)$ & 0.10 \\
\hline Comorbidities & 126(6.3) & $24(4.9)$ & 102(6.7) & 0.15 & $50(5.9)$ & $52(7.9)$ & 0.12 \\
\hline Cardiovascular diseases & $64(3.2)$ & $9(1.8)$ & $55(3.6)$ & 0.052 & $27(3.2)$ & $28(4.2)$ & 0.27 \\
\hline liver and kidney diseases & $14(0.7)$ & $2(0.4)$ & $12(0.8)$ & 0.57 & $4(0.5)$ & $8(1.2)$ & 0.19 \\
\hline Others & $48(2.4)$ & $13(2.7)$ & $35(2.3)$ & 0.65 & $19(2.2)$ & $16(2.4)$ & 0.80 \\
\hline Shortage of supplies & $444(22.2)$ & 106(21.8) & $338(22.3)$ & 0.80 & 173(20.3) & $165(25.0)$ & 0.12 \\
\hline Burden of caring for the elderly or children & $1177(58.8)$ & 265(54.4) & $912(60.2)$ & 0.02 & 494(57.9) & 418(63.2) & 0.04 \\
\hline Family infected with COVID-19 & $301(15.0)$ & 75(15.4) & 226(14.9) & 0.80 & 126(14.8) & $100(15.1)$ & 0.85 \\
\hline COVID-related bereavement & 164(8.2) & $40(8.2)$ & 124(8.2) & 0.99 & $51(6.0)$ & 73(11.0) & $<0.01$ \\
\hline
\end{tabular}

BMI, body mass index; COVID-19, Corona Virus Disease 2019.

Continuous variables are presented as median (IQR) (Mann-Whitney test). Categorical variables are expressed as number (percentages) (Chi-square or Yates' continuity corrected chisquare test). ${ }^{a} P$ values indicate differences between non-medical staff and medical staff. ${ }^{b} P$ values indicate differences between non-frontline and frontline medical workers. $P<0.05$ was considered statistically significant. 

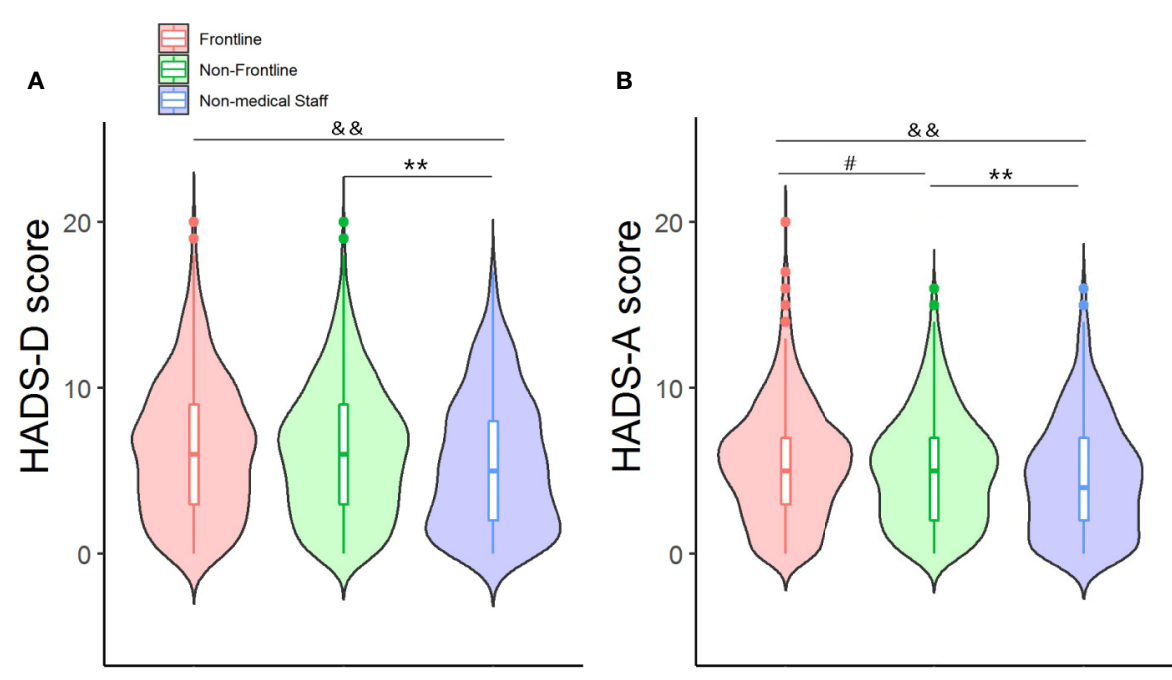

FIGURE 1 | Frontline medical staff mainifested higher HADS-A (A) and HADS-D (B) scores compared to non-frontline and non-medical staff. Data are presented as median (interquartile range). ${ }^{\#} p<0.05$, Frontline vs Non-frontline medical staff; ${ }^{* *} \mathrm{p}<0.01$, Non-frontline medical staff vs Non-medical staff; ${ }^{\& \&} \mathrm{p}<0.01$ Frontline medical staff vs Non-medical staff.
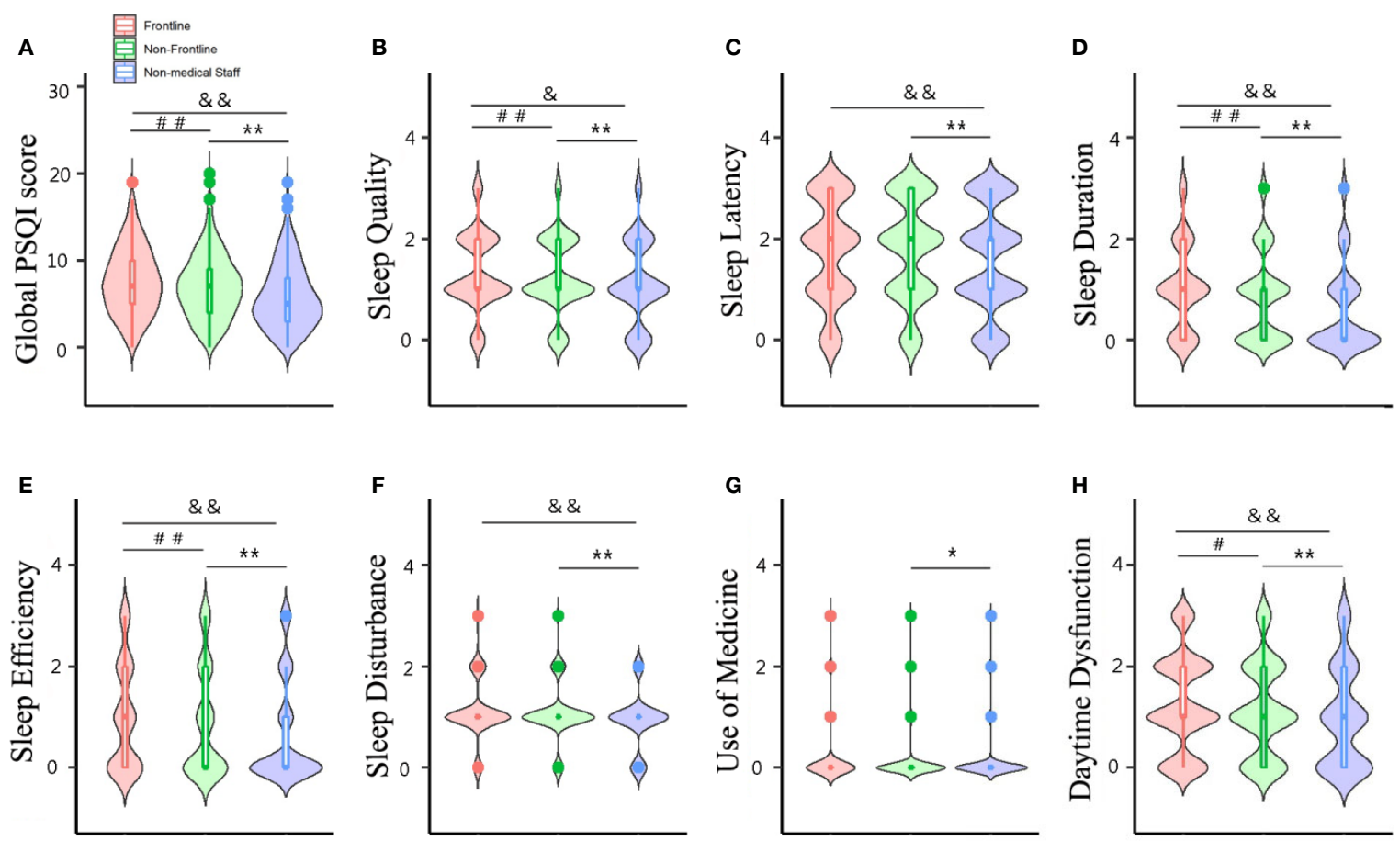

FIGURE 2 | Global PSQI score (A) and component scores (B-H) of the participants. Data are presented as median (interquartile range). ${ }^{\# \#} \mathrm{p}<0.01, " \# \mathrm{p}<0.05$, Frontline vs Non-frontline medical staff; ${ }^{* *} p<0.01,{ }^{*} p<0.05$, Non-frontline medical staff vs Non-medical staff; ${ }^{\&} \mathrm{p} p<0.01,{ }^{{ }^{*}} p<0.05$, Frontline medical staff vs Nonmedical staff.

no significant difference in median component scores of sleep medication use between those working directly with COVID-19 patients and other subgroups (Figure 2G). The medical staff were more vulnerable to poor sleep during the COVID-19 epidemic
(Table 2), while anxiety and depression were prevalent in the entire cohort (Table 2). Among the healthcare workers, 1,000 (66.1\%) reported poor sleep quality (PSQI>5), of which 301(19.9\%) reported very poor sleep quality (PSQI $>10)$. Compared to the non-frontline 
TABLE 2 | Incidence of poor sleep quality, anxiety, and depression in overall cohort and subgroups.

\begin{tabular}{|c|c|c|c|c|c|c|c|c|}
\hline & & \multirow[t]{2}{*}{ Overall cohort $(n=2$ 001) } & \multicolumn{2}{|c|}{ Medical staff } & \multirow[t]{2}{*}{$\mathbf{P}^{\mathrm{a}}$ value } & \multicolumn{2}{|c|}{ Frontline medical workers } & \multirow[t]{2}{*}{$\mathbf{P}^{\mathrm{b}}$ value } \\
\hline & & & $\mathrm{No}(\mathrm{n}=487)$ & Yes(n=1514) & & No $(n=853)$ & Yes $(n=661)$ & \\
\hline \multicolumn{9}{|c|}{ Overall age } \\
\hline & PSQI>5 & 1233 (61.6) & 233(47.8) & $1000(66.1)$ & $<0.01$ & $550(64.5)$ & $450(68.1)$ & 0.14 \\
\hline & $>10$ & 366 (18.3) & 65(13.3) & 301(19.9) & $<0.01$ & $145(17.0)$ & 156(23.6) & $<0.01$ \\
\hline & HADS-A>7 & 452 (22.6) & $98(20.1)$ & $354(23.4)$ & 0.13 & 190(22.3) & $164(24.8)$ & 0.25 \\
\hline & HADS-D>7 & 701 (35.0) & 155(31.8) & $546(36.1)$ & 0.09 & $304(35.6)$ & 242(36.6) & 0.70 \\
\hline \multicolumn{9}{|c|}{ Age range, y } \\
\hline $18-35$ & & 1213 & 335 & 878 & & 515 & 363 & \\
\hline & PSQI>5 & $701(57.8)$ & 144(43.0) & $557(63.4)$ & $<0.01$ & $313(60.8)$ & $244(67.2)$ & 0.051 \\
\hline & $>10$ & 186(15.3) & 29(8.7) & 157(17.9) & $<0.01$ & 71(13.8) & $86(35.2)$ & $<0.01$ \\
\hline & HADS-A>7 & 234(19.3) & $62(18.5)$ & 172(19.6) & 0.67 & $95(18.4)$ & $77(11.6)$ & 0.31 \\
\hline & HADS-D>7 & $391(32.2)$ & $101(30.1)$ & 290(33.0) & 0.34 & 169(32.8) & $121(33.3)$ & 0.87 \\
\hline $36-44$ & & 480 & 93 & 387 & & 199 & 188 & \\
\hline & PSQI>5 & $325(67.7)$ & $57(61.3)$ & 268(69.3) & 0.14 & 138(69.3) & $130(69.1)$ & 0.97 \\
\hline & $>10$ & 102(21.3) & 23(24.7) & $79(20.4)$ & 0.36 & $35(17.6)$ & 44(23.4) & 0.16 \\
\hline & HADS-A>7 & $130(27.1)$ & $26(28.0)$ & 104(26.9) & 0.83 & $50(25.1)$ & $54(28.7)$ & 0.42 \\
\hline & HADS-D>7 & $202(42.1)$ & $42(45.2)$ & $160(41.3)$ & 0.50 & $77(38.7)$ & $83(44.1)$ & 0.28 \\
\hline $45-65$ & & 308 & 59 & 249 & & 139 & 110 & \\
\hline & PSQI>5 & 207(67.2) & $32(54.2)$ & 175(70.3) & 0.02 & $99(71.2)$ & 76(69.1) & 0.71 \\
\hline & $>10$ & $78(25.3)$ & 13(22.0) & $65(26.1)$ & 0.52 & $39(28.1)$ & 26(23.6) & 0.43 \\
\hline & HADS-A $>7$ & 88(28.6) & 10(16.9) & 78(31.3) & 0.03 & 45(32.4) & 33(30.0) & 0.69 \\
\hline & HADS-D>7 & $108(35.1)$ & 12(20.3) & 96(38.6) & $<0.01$ & $58(41.7)$ & $38(34.5)$ & 0.25 \\
\hline
\end{tabular}

PSQI, Pittsburgh Sleep Quality Index; HASD-A, Hospital Anxiety and Depression Scale-Anxiety; HASD-D, Hospital Anxiety and Depression Scale-Depression.

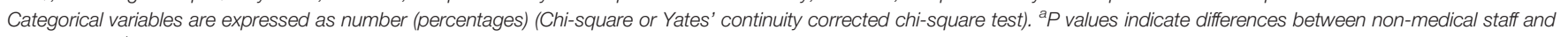
medical staff. ${ }^{b} \mathrm{P}$ values indicate differences between non-frontline and frontline medical workers. $P<0.05$ was considered statistically significant.

workers, those in frontline were more likely to experience very poor sleep (156[23.6\%] vs $145[17 \%])$. In addition, $354(23.4 \%)$ had anxiety (HADS-A>7), and 546 (36.1\%) suffered from depression (HADS$\mathrm{D}>7$ ). No significant difference was seen in the prevalence of anxiety or depression between the frontline and non-frontline healthcare workers. (Table 2). Medical staff aged 18-35 (comprised of 63.4\% and $17.9 \%$ of individuals with poor and very poor sleep quality) were more prone to develop sleep disorders than the general public (comprised of $43.0 \%$ and $8.7 \%$ of poor and very poor sleep quality). Serious sleep problems were more common in young nurses and doctors who had direct contact with infected patients than others in the same age groups (86/363 (35.2\%) vs 71/515 [13.8\%]) (Table 2). Interestingly, the incidence of anxiety and depression in medical staff aged $45-65$ were up to $31.3 \%$ and $38.6 \%$, respectively, almost twice as much of age-matched non-medical staff (16.9\% and $20.3 \%$, respectively) (Table 2). However, there was no significant difference in the prevalence of sleep disorders, anxiety or depression between healthcare workers aged 36-44 and their peers (Table 2).

\section{Risk Factors for Poor Sleep Quality}

Binary logistic regression showed that male sex, education, smoking, alcohol consumption, BMI, geographical region, and novel coronavirus infection were not predictive of poor sleep quality (Table 3). However, age over 35 years, married, comorbidities, medical job, shortage of daily necessities, fostering or parenting pressure, death of a family member, anxiety, and depression were associated with poor sleep quality (Table 3 ). The multivariable analysis indicated that medical personnel, burden of caring for the elderly or children, bereavement, and anxiety-depression were independent risk factors for sleep problems (Table 3).

\section{DISCUSSION}

We analyzed the sleep quality, anxiety and depression in 1,514 medical workers and 487 non-medical workers from Mar 4 to 9, 2020 , and found that $61.6 \%$ of the respondents had sleep problems, $22.6 \%$ felt anxiety, and 35\% suffered from depression. Frontline healthcare workers were more prone to severe sleep problems compared to the non-frontline and non-healthcare workers, while no significant differences were seen in the prevalence of anxiety or depression among the three subgroups. Frontline medical staff, non-frontline medical staff, parental burden, COVID-related bereavement, anxiety and depression were the independent risk factors of poor sleep quality.

The COVID-19 outbreak is the third major global public health event caused by coronavirus after SARS and Middle East respiratory syndrome (MERS). Studies show that $21-57 \%$ of the medical personnel experienced emotional distress during the SARS outbreak (22-24). Psychiatric morbidity was also observed in almost $51 \%$ of the healthcare workers during the MERS outbreak (25). During the early stage of the COVID- 19 outbreak, $28.8 \%$ of the general population in China reported anxiety symptoms, and $16.5 \%$ reported depressive symptoms (26). However, the incidence of anxiety, depression and insomnia among the healthcare workers was $44.6 \%, 50.4 \%$ and $34.0 \%$ respectively (18). We found that anxiety, depression and sleep disturbance respectively occurred in $20.1 \%, 31.8 \%$ and $47.8 \%$ of the general population, and the incidence was higher among health professionals at $23.4 \%, 36.1 \%$ and $66.1 \%$ respectively. In addition, a previous study reported that $39.2 \%$ of the medical staff in China suffer from sleep disturbances (17). Nevertheless, positive changes in the COVID-19 situation 
TABLE 3 | Binary logistic regression of risk factors for poor sleep quality during COVID-19 outbreak.

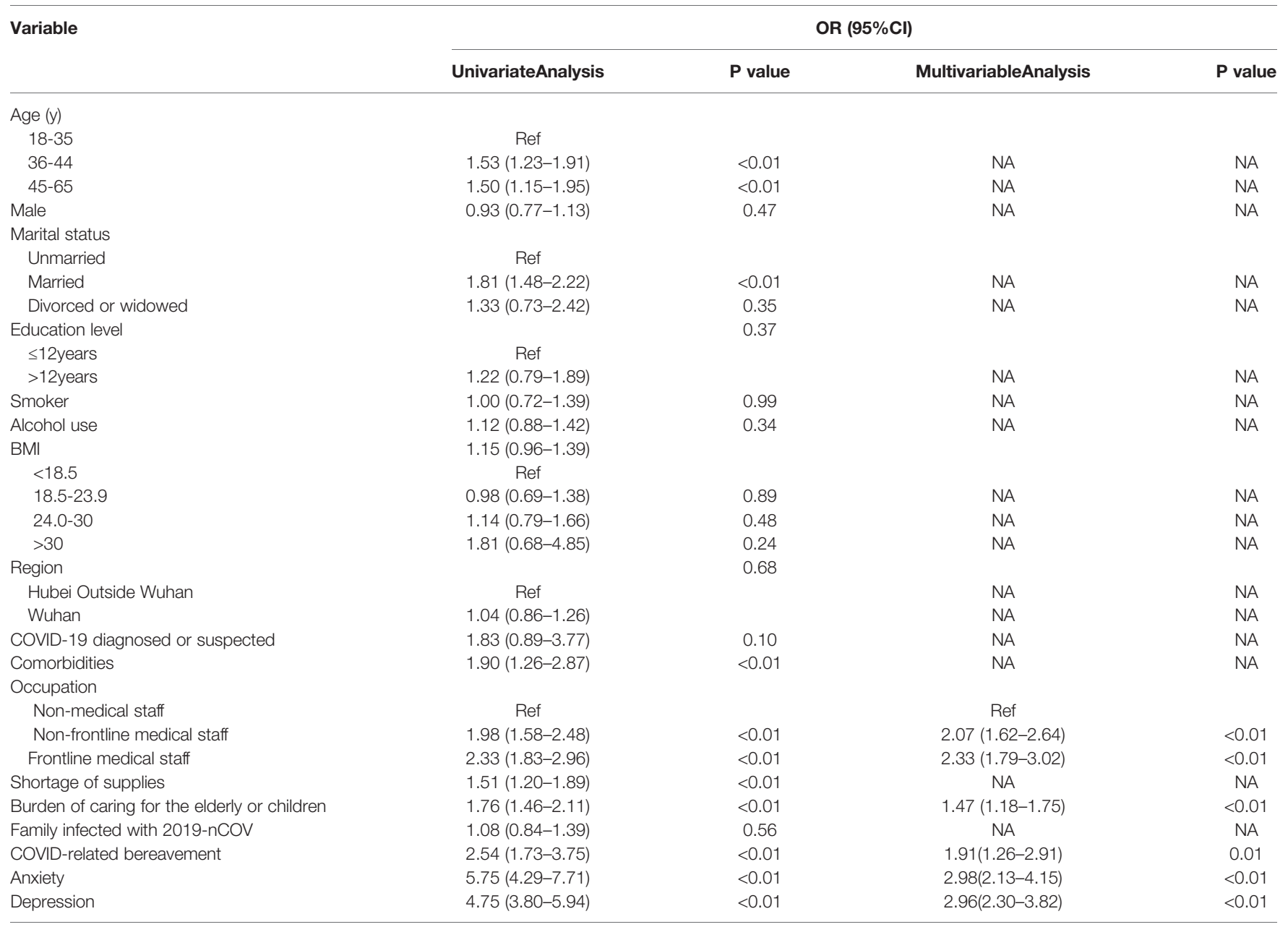

BMI, body mass index; COVID-19, Corona Virus Disease 2019; OR, odds ratio; Ref, reference; NA, not applicable.

might alleviate the negative emotions among the healthcare workers in Hubei Province.

The sleep quality was poorer in the frontline medical staff compared to the non-frontline and non-medical cohorts. The mean PSQI scores of SARS vs non-SARS unit nurses at the beginning of the SARS outbreak were 7.2 and 4 respectively (27). A survey on 116 Taiwan nurses showed that sleep quality was worse (average total PSQI score 12) before caring for SARS patients, and continued to be poor (average global PSQI score 8-10) until 3 months afterwards (28). Another survey concluded that mean PSQI scores of SARS unit nurses ranged from 7.2 to 5.2 during a 7-week period (27). Single-center evidence from the early COVID-19 outbreak period indicated that the sleep quality of frontline healthcare personnel was lower with a mean PSQI score of 8.6, compared with normal Chinese residents (29). In our study, the median PSQI scores of both frontline and non-frontline workers were 7 , although frontline medical workers were more susceptible to severe sleep disturbances. During the SARS outbreak, 14 to $37 \%$ of the frontline staff complained of insomnia compared to 5 to $9.7 \%$ of non-frontline workers $(27,30)$. Bai $\mathrm{Y}$ et al. found that the incidence of anxiety-depression was similar between the frontline healthcare workers and administrative staff during a survey conducted from
May 29 to June 5, 2003 (28). Consistent with this, anxiety and depression were not significantly different among the three subgroups in our study. This could be attributed to policies for addressing these mental health problems, such as identifying infection as work-related injury, assistance from other provinces, a shift system to allow allied staff to rest, additional salaries or subsidies and psychological intervention (10). Furthermore, the stress due to quarantine, worrying about family members etc. affect both healthcare and non-healthcare workers, and are as significant as medical stressors.

Binary logistic regression analysis identified medical occupation, parental burden, death of a loved one, anxiety and depression as the 5 factors of poor sleep quality. During the SARS outbreak as well, heavy workload, family stress, personal or family lifestyle and negative feelings were significantly correlated with insomnia $(23,27,31)$. Studies have also indicated that high anxiety level correlate with sleep quality $(32,33)$, and a similar association was found in our survey as well. In line with a previous study describing the relationship between depressive symptoms and sleep disturbance (34), allied healthcare workers who suffered from depression were 2.96 (95\% CI 2.30-3.82) times more likely to have disturbed sleep compared to those lacking depressive 
symptoms. Furthermore, sleep problems, especially insomnia, have been bidirectionally implicated in the development of mood disturbances, indicating that insomnia may be a predictor of anxiety and depression, and vice-versa (35). In the case of Chinese physicians, a cross-sectional study concluded that participants who had insufficient sleep ( $<6 \mathrm{~h}$ per day) were more predisposed to anxiety (OR $=2.70,95 \% \mathrm{CI}$ : $1.51-4.83)$ and depression ( $\mathrm{OR}=1.58,95 \% \mathrm{CI}: 0.95-2.64)$ than those who had proper sleep ( $\geq 8 \mathrm{~h}$ per day) (36). Accordingly, effective amelioration of sleep disorders may help abate the occurrence of subsequent or comorbid mood disturbances and vice-versa (37). There were some limitations in our study that ought to be addressed.

First, an online questionnaire cannot guarantee accurate demographic or other information from the respondents (19). Second, apart from data about shift workers, we did not dynamically observe the psychological impact of COVID-19 outbreak on healthcare professionals. Our survey was conducted when the measures taken for controlling COVID-19 outbreak were showing positive results, which may have affected the psychological status of the respondents since they adapted to coronavirus characterized by high transmissibility but low fatality rates. Third, the questionnaire used for the survey did not include other mental illnesses such as fear, denial, anger and PTSD, since some frontline workers were very reluctant to answer all 57 questions. Moreover, it may be impossible to overcome the selection bias due to the study being based on an online survey. Of the 2,001 respondents in the present study, an overwhelming majority were young females. Ultimately, PSQI was found to be frequently associated with the evaluation of daytime sleepiness therefore it may be useful to add the evaluation of the Epworth sleepiness scale (38). In addition, anxiety and depression cannot be diagnosed by only using questionnaires, therefore further evidence through a psychological evaluation will be required.

\section{REFERENCES}

1. Zhu N, Zhang D, Wang W, Li X, Yang B, Song J, et al. A novel coronavirus from patients with pneumonia in China, 2019. N Engl J Med (2020) 382 (8):727-33. doi: 10.1056/NEJMoa2001017

2. World Health Organization. Naming the coronavirus disease (COVID-19) and the virus that causes it (2020). Available at: https://www.who.int/emergencies/ diseases/novel-coronavirus-2019/technical-guidance/naming-thecoronavirus-disease-(covid-2019)-and-the-virus-that-causes-it (Accessed Feb 11, 2020).

3. Li X, Zai J, Wang X, Li Y. Potential of large "first generation" human-tohuman transmission of 2019-nCoV. J Med Virol (2020) 92(4):448-54. doi: 10.1002/jmv.25693

4. Bai Y, Yao L, Wei T, Tian F, Jin DY, Chen L, et al. Presumed asymptomatic carrier transmission of COVID-19. JAMA (2020) 323(14):1406-7. doi: 10.1001/ jama.2020.2565

5. Paules CI, Marston HD, Fauci AS. Coronavirus infections-more than just the common cold. JAMA (2020) 323(8):707-8. doi: 10.1001/jama.2020.0757

6. National Health Commission of the People's Republic of China. Latest developments in epidemic control on March 8 (2020). Available at: http:// www.nhc.gov.cn/xcs/s3574/202003/a54a40ae28764f3581f36cc31204433c. shtml (Accessed Mar 9, 2020).

7. National Health Commission of the People's Republic of China. Handbook of Prevention and Treatment of the Pneumonia Caused by the Novel Coronavirus

\section{CONCLUSIONS}

The COVID-19 outbreak has affected the sleep quality of healthcare workers. As the epidemic evolves and psychological interventions are optimized, the physical and mental fitness of the frontline staff may improve. Long-term psychosocial effects of COVID-19 on allied healthcare workers deserve further investigation to prepare for any future public health disasters.

\section{DATA AVAILABILITY STATEMENT}

The original contributions presented in the study are included in the article/supplementary material; further inquiries can be directed to the corresponding authors.

\section{ETHICS STATEMENT}

The studies involving human participants were reviewed and approved by: The ethic committee of Renmin Hospital of Wuhan University. Written informed consent to participate in this study was provided by the participants' legal guardian/next of kin.

\section{AUTHOR CONTRIBUTIONS}

WW, SW, XZ, HY, and LS designed the study. TL and HJ recruited the respondents. WW and SW collected and analyzed psychological data. WW and SW drafted the manuscript. XZ and LS undertook a critical revision of the manuscript. All authors contributed to the article and approved the submitted version.

(2019-nCoV). Available at: http://en.nhc.gov.cn/2020-02/06/c_76726.htm (Accessed Feb 6, 2020).

8. Wu Z, McGoogan JM. Characteristics of and important lessons from the coronavirus disease 2019 (COVID-19) outbreak in China: summary of a report of 72314 cases from the Chinese center for disease control and prevention. JAMA (2020) 323(13):1239-42. doi: 10.1001/jama.2020.2648

9. Guan WJ, Ni ZY, Hu Y, Liang WH, Ou CQ, He JX, et al. Clinical characteristics of coronavirus disease 2019 in China. N Engl J Med (2020) 382(18):1708-20. doi: 10.1056/NEJMoa2002032

10. Kang L, Li Y, Hu S, Chen M, Yang C, Yang BX, et al. The mental health of medical workers in Wuhan, China dealing with the 2019 novel coronavirus. Lancet Psychiatry (2020) 7(3):e14. doi: 10.1016/S2215-0366(20)30047-X

11. McAlonan GM, Lee AM, Cheung V, Cheung C, Tsang KW, Sham PC, et al. Immediate and sustained psychological impact of an emerging infectious disease outbreak on health care workers. Can J Psychiatry (2007) 52(4):241-7. doi: 10.1177/070674370705200406

12. Wu P, Fang Y, Guan Z, Fan B, Kong J, Yao Z, et al. The psychological impact of the SARS epidemic on hospital employees in China: exposure, risk perception, and altruistic acceptance of risk. Can J Psychiatry (2009) 54 (5):302-11. doi: 10.1177/070674370905400504

13. Li L, Wan C, Ding R, Liu Y, Chen J, Wu Z, et al. Mental distress among Liberian medical staff working at the China Ebola Treatment Unit: a cross sectional study. Health Qual Life Outcomes (2015) 13:156. doi: 10.1186/ s12955-015-0341-2 
14. Huang $\mathrm{Y}$, Zhao N. Generalized anxiety disorder, depressive symptoms and sleep quality during COVID-19 outbreak in China: a web-based cross-sectional survey. Psychiatry Res (2020) 288:112954. doi: 10.1016/j.psychres.2020.112954

15. Forte G, Favieri F, Tambelli R, Casagrande M. COVID-19 Pandemic in the Italian Population: Validation of a Post-Traumatic Stress Disorder Questionnaire and Prevalence of PTSD Symptomatology. Int J Environ Res Public Health (2020) 17(11):E4151. doi: 10.3390/ijerph17114151

16. Chen Q, Liang M, Li Y, Guo J, Fei D, Wang L, et al. Mental health care for medical staff in China during the COVID-19 outbreak. Lancet Psychiatry (2020) 7(4):e15-6. doi: 10.1016/S2215-0366(20)30078-X

17. Qiu D, Yu Y, Li RQ, Li YL, Xiao SY. Prevalence of sleep disturbances in Chinese healthcare professionals: a systematic review and meta-analysis. Sleep Med (2020) 67:258-66. doi: 10.1016/j.sleep.2019.01.047

18. Hinz A, Glaesmer H, Brähler E, Löffler M, Engel C, Enzenbach C, et al. Sleep quality in the general population: psychometric properties of the Pittsburgh Sleep Quality Index, derived from a German community sample of 9284 people. Sleep Med (2017) 30:57-63. doi: 10.1016/j.sleep.2016.03.008

19. The State Council The People's Republic of China. Intl cooperation stressed in epidemic control and prevention. Available at: http://english.www.gov.cn/ premier/news/202003/09/content_WS5e66433bc6d0c201c2cbde3e.html (Accessed Mar 9, 2020).

20. Zigmond AS, Snaith RP. The hospital anxiety and depression scale. Acta Psychiatr Scand (1983) 67(6):361-70. doi: 10.1111/j.1600-0447.1983.tb09716.x

21. Sim K, Chua HC. The psychological impact of SARS: a matter of heart and mind. CMAJ (2004) 170(5):811-2. doi: 10.1503/cmaj.1032003

22. Nickell LA, Crighton EJ, Tracy CS, Al-Enazy H, Bolaji Y, Hanjrah S, et al. Psychosocial effects of SARS on hospital staff: survey of a large tertiary care institution. CMAJ (2004) 170(5):793-8. doi: 10.1503/cmaj.1031077

23. Tam CW, Pang EP, Lam LC, Chiu HF. Severe acute respiratory syndrome (SARS) in Hong Kong in 2003: stress and psychological impact among frontline healthcare workers. Psychol Med (2004) 34(7):1197-204. doi: $10.1017 / \mathrm{s} 0033291704002247$

24. Lee SM, Kang WS, Cho AR, Kim T, Park JK. Psychological impact of the 2015 MERS outbreak on hospital workers and quarantined hemodialysis patients. Compr Psychiatr (2018) 87:123-7. doi: 10.1016/j.comppsych.2018.10.003

25. Wang C, Pan R, Wan X, Tan Y, Xu L, Ho CS, et al. Immediate psychological responses and associated factors during the initial stage of the 2019 coronavirus disease (covid-19) epidemic among the general population in China. Int J Environ Res Public Health (2020) 17(5):1729. doi: 10.3390/ ijerph17051729

26. Su TP, Lien TC, Yang CY, Su YL, Wang JH, Tsai SL, et al. Prevalence of psychiatric morbidity and psychological adaptation of the nurses in a structured SARS caring unit during outbreak: a prospective and periodic assessment study in Taiwan. J Psychiatr Res (2007) 41(1-2):119-30. doi: 10.1016/ j.jpsychires.2005.12.006

27. Chen R, Chou KR, Huang YJ, Wang TS, Liu SY, Ho LY. Effects of a SARS prevention programme in Taiwan on nursing staff's anxiety, depression and sleep quality: a longitudinal survey. Int J Nurs Stud (2006) 43(2):215-25. doi: 10.1016/j.ijnurstu.2005.03.006
28. Xiao H, Zhang Y, Kong D, Li S, Yang N. The effects of social support on sleep quality of medical staff treating patients with coronavirus disease 2019 (COVID-19) in January and February 2020 in China. Med Sci Monit (2020) 26:e923549. doi: 10.12659/MSM.923549

29. Bai Y, Lin CC, Lin CY, Chen JY, Chue CM, Chou P. Survey of stress reactions among health care workers involved with the SARS outbreak. Psychiatr Serv (2004) 55(9):1055-7. doi: 10.1176/appi.ps.55.9.1055

30. Lau JT, Yang X, Pang E, Tsui HY, Wong E, Wing YK. SARS-related perceptions in Hong Kong. Emerg Infect Dis (2005) 11(3):417-24. doi: 10.3201/eid1103.040675

31. Brown WJ, Wilkerson AK, Boyd SJ, Dewey D, Mesa F, Bunnell BE. A review of sleep disturbance in children and adolescents with anxiety. J Sleep Res (2018) 27(3):e12635. doi: 10.1111/jsr.12635

32. Gould CE, Spira AP, Liou-Johnson V, Cassidy-Eagle E, Kawai M, Mashal N, et al. Association of anxiety symptom clusters with sleep quality and daytime sleepiness. J Gerontol B Psychol Sci Soc Sci (2018) 73(3):413-20. doi: 10.1093/ geronb/gbx020

33. Guo L, Deng J, He Y, Deng X, Huang J, Huang G, et al. Prevalence and correlates of sleep disturbance and depressive symptoms among Chinese adolescents: a cross-sectional survey study. BMJ Open (2014) 4(7):e005517. doi: 10.1136/bmjopen-2014-005517

34. Wright Kevin B. Researching Internet-Based Populations: Advantages and Disadvantages of Online Survey Research, Online Questionnaire Authoring Software Packages, and Web Survey Services. J Comput Mediat Commun (2005) 10. doi: 10.1111/j.1083-6101.2005.tb00259.x

35. Alvaro PK, Roberts RM, Harris JK. A Systematic Review Assessing Bidirectionality between Sleep Disturbances, Anxiety, and Depression. Sleep (2013) 36(7):1059-68. doi: 10.5665/sleep.2810

36. Gong Y, Han T, Chen W, Dib HH, Yang G, Zhuang R, et al. Prevalence of anxiety and depressive symptoms and related risk factors among physicians in China: a cross-sectional study. PloS One (2014) 9(7):e103242. doi: 10.1371/ journal.pone. 0103242

37. Kaneita Y, Yokoyama E, Harano S, Tamaki T, Suzuki H, Munezawa T, et al. Associations between sleep disturbance and mental health status: a longitudinal study of Japanese junior high school students. Sleep Med (2009) 10(7):780-6. doi: 10.1016/j.sleep.2008.06.014

38. Johns MW. A new method for measuring daytime sleepiness: the Epworth sleepiness scale. Sleep (1991) 14(6):540-5. doi: 10.1093/sleep/14.6.540

Conflict of Interest: The authors declare that the research was conducted in the absence of any commercial or financial relationships that could be construed as a potential conflict of interest.

Copyright (๑) 2020 Wang, Song, Xia, He, Tang, Hou and Lei. This is an open-access article distributed under the terms of the Creative Commons Attribution License (CC $B Y)$. The use, distribution or reproduction in other forums is permitted, provided the original author(s) and the copyright owner(s) are credited and that the original publication in this journal is cited, in accordance with accepted academic practice. No use, distribution or reproduction is permitted which does not comply with these terms. 